Supporting Information

\title{
Oxychalcogenide BaGeOSe 2 : Highly Distorted Mixed-Anion Building Units Leading to a Large Second-Harmonic Generation Response
}

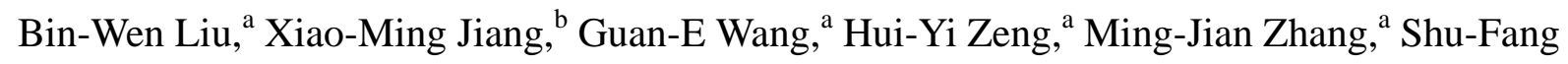
Li, $^{\text {a }}$ Wei-Huan Guo, ${ }^{\text {a }}$ Guo-Cong Guo ${ }^{\text {a }}$

${ }^{a}$ State Key Laboratory of Structural Chemistry, Fujian Institute of Research on the Structure of Matter, Chinese Academy of Sciences, Fuzhou, Fujian 350002, P. R. China

${ }^{\mathrm{b}}$ Institute for Quantum Materials, Hubei Polytechnic University, Huangshi 435003, P. R. China 


\section{Supporting Information Index}

\section{Experimental Section}
1) Syntheses
2) X-ray crystallography
3) Energy-Dispersive X-ray Spectroscopy (EDS) and Inductively Coupled Plasma (ICP) Analysis
4) Thermal Analysis
5) UV-Vis-NIR Diffuse Reflectance, IR and Raman Spectroscopy.
6) Second-Harmonic Generation (SHG) Measurements
7) Computation Procedure

\section{Figures and Tables}

Figure S1. Photo of a medium-size crystal of $\mathbf{1}$ grown using the facile synthetic method.

Figure S2. EDX analysis of 1.

Figure S3. Experimental and simulated powder X-ray diffraction data of $\mathbf{1}$.

Figure S4. DSC curves reveal the melting and recrystallization events of $\mathbf{1}$.

Figure S5. PXRD of the pristine material and the sample obtained after DTA.

Figure S6. UV-Vis diffuse reflectance spectra of 1.

Figure S7. IR spectrum of 1 .

Figure S8. Raman spectra of $\mathbf{1}$.

Figure S9. Band structures of $\mathbf{1}$.

Figure S10. Energy dependences of the real part $\varepsilon_{1}$ of $\mathbf{1}$.

Figure S11. Energy dependences of the imaginary part $\varepsilon_{2}$ of $\mathbf{1}$.

Figure S12. Variations of the calculated refractive index $n$ of $\mathbf{1}$.

Figure S13. Calculated birefringence $\Delta n$ of 1 .

Figure S14. Calculated frequency-dependent SHG coefficients of 1.

Table S1. Crystal Data and Structure Refinements for 1.

Table S2. Selected Bond Distances ( $)$ and Angles (deg) in $\mathbf{1}$.

\section{References.}




\section{Experimental section:}

\section{Syntheses}

All starting reactants were handled inside an argon-filled glovebox with controlled oxygen and moisture levels below $0.1 \mathrm{ppm}$. Ba metal (99.5\%), Se powder (99.9\%), $\mathrm{GeO}_{2}$ powder $(99.5 \%)$, and KI powder $(99.5 \%)$ were used as obtained without further purification. For synthesizing title compound, a stoichiometric mixture with the starting materials $\mathrm{Ba}$, $\mathrm{GeO}_{2}$, Se, B and extra $\mathrm{KI}$ as a flux in molar ratio 3:3:6:2:15 were loaded into quartz tubes and then flame-sealed under vacuum about $10^{-4}$ Torr. The samples were placed in a temperature-controlled muffle furnace, held at $300{ }^{\circ} \mathrm{C}$ for $5 \mathrm{~h}$, then heated to $700{ }^{\circ} \mathrm{C}$ in $5 \mathrm{~h}$ and dwelled for 4 days, finally cooled down to $350{ }^{\circ} \mathrm{C}$ before the furnace power was switched off. Thus, colorless single crystals of the title compound were obtained in high-yield of $95 \%$ (based on $\mathrm{Ba}$ ) after washing with distilled water, and they were stable in air and moisture conditions for several months. Boron chalcogen have been found to be convenient agents for the syntheses of a broad range of metal chalcogenides from the corresponding metal oxides, in which the element $\mathrm{B}$ can act as a reducing reagent to extract oxygen from metal oxides due to its strong oxygen affinity in the reactive system. It should be noted that several attempts to synthesize compound $\mathbf{1}$ with the same molar ratio of starting materials but without element B have been made, but all were failure. These observations indicate that the element B can play a reaction promoter role for the formation of title compound. Therefore, the equation for the reaction in this synthesize procedures is

$$
3 \mathrm{Ba}+3 \mathrm{GeO}_{2}+6 \mathrm{Se}+2 \mathrm{~B}+15 \mathrm{KI} \rightarrow 3 \mathrm{BaGeOSe}_{2}+\mathrm{B}_{2} \mathrm{O}_{3}+15 \mathrm{KI} .
$$

\section{X-ray crystallography}




\subsection{Single-Crystal X-ray Diffraction (XRD)}

Single-crystal XRD data were performed on a Rigaku Pilatus CCD diffractometer equipped with a graphite-monochromated Mo K $\alpha$ radiation $(\lambda=0.71073 \AA)$ at $293 \mathrm{~K}$. The intensity data sets were collected using a $\omega$-scan technique and reduced using Crystalclear software. ${ }^{1}$ The structures were solved by direct methods and refined by full-matrix least-squares methods on $F^{2}$ with anisotropic thermal parameters for all atoms. All of the calculations were performed with the Siemens SHELXTL, version 5, package of crystallographic software. ${ }^{2}$ The space group was determined to be $P 2{ }_{1} 2_{1} 2_{1}$, based on the intensity distribution and systematic absences of the observed structure factors. The final structure was examined for additional symmetry with ADDSYM/PLATON. ${ }^{3}$ The program STRUCTURE TIDY was used to put the positional parameters in a standard setting. ${ }^{4}$ Additional crystallographic details are given in Table S1, further information may be found in the Supporting Information.

\subsection{Powder XRD}

The powder XRD data of $\mathbf{1}$ were recorded on an automated Rigaku MiniFlex II X-ray diffractometer equipped with a diffracted monochromator set for $\mathrm{Cu} K \alpha$ radiation $(\lambda=$ $1.54057 \AA$ ) , operating at $30 \mathrm{kV} / 40 \mathrm{~mA}$.

\section{EDX and Inductively Coupled Plasma (ICP) Measurements}

Semiquantitative EDX was performed with an EDX-equipped Hitachi S-3500 scanning electron microscopy spectrometer on the crystals which had been measured by single-crystal $\mathrm{XRD}$, gives the $\mathrm{Ba} / \mathrm{Ge} / \mathrm{Se}$ molar ratio of 1.1:1.0:2.0. Meanwhile, ICP had been investigated on Ultima 2 inductively coupled plasma OES spectrometer, showing that the ratio of 
$\mathrm{Ba} / \mathrm{Ge} / \mathrm{Se}$ is $1.0: 1.1: 2.0$. Both the EDS and ICP results are in according with the formula based on the single crystal XRD study.

\section{Thermal Analysis}

The thermal properties of $\mathbf{1}$ were performed by differential scanning calorimetry (DSC) analysis using a TGA-DSC-1-1000 (STARE) thermal analyzer. A sample ( $20 \mathrm{mg}$ ) of ground crystalline material was sealed in a silica ampule. The sample was heated to $1000{ }^{\circ} \mathrm{C}$ at $10{ }^{\circ} \mathrm{C} \cdot \mathrm{min}^{-1}$, and then it was cooled to $25^{\circ} \mathrm{C}$ at a rate of $10{ }^{\circ} \mathrm{C} \cdot \mathrm{min}^{-1}$. The products of the DSC experiment were examined by powder XRD. Reproducibility of the results was confirmed by running multiple heating/cooling cycles.

\section{UV-Vis-Near-IR (NIR) Diffuse Reflectance, Infrared (IR) and Raman Spectroscopy.}

The optical diffuse reflectance spectrum of $\mathbf{1}$ was measured at room temperature using a PerkinElmer Lambda 900 UV-vis spectrophotometer equipped with an integrating sphere attachment and $\mathrm{BaSO}_{4}$ as reference. The absorption spectra were calculated from reflection spectra using the Kubelka-Munk function: $\alpha / S=(1-R)^{2} / 2 R,{ }^{5}$ in which $\alpha$ is the absorption coefficient, $S$ is the scattering coefficient, and $R$ is the reflectance. IR spectra were recorded using a Nicolet Magana 750 Fourier transform-IR spectrophotometer in the range 4000-400

$\mathrm{cm}^{-1}$. Powdery samples were diluted with dry $\mathrm{KBr}$ and pressed into pellets. Raman spectra were collected of ground crystalline samples on a Labram HR800 Evolution. The beam source was emitted from a diode laser with $785 \mathrm{~nm}$ radiation.

\section{Second-harmonic generation (SHG) measurement}

The powder SHG measurements of $\mathbf{1}$ were investigated using a modified Kurtz-Perry powder technique. The incident laser of $1400 \mathrm{~nm}$ was applied because there were some 
absorptions below $700 \mathrm{~nm}$ (see Figure S6). Microcrystalline sample were sieved into several distinct particle size ranges $(30-50,50-75,75-100,100-150,150-200,200-250$ and 250-300 $\mu \mathrm{m}$ ) for the SHG phase-match measurements. The crystalline sample of $\mathrm{AgGaS}_{2}$ of similar particle size served as the standard. The doubled frequency signals were detected by an Andor DU420A-BR-DD CCD after the mixed signals passed through the monochromator.

\section{Computation Procedure}

Calculations of electronic band structures were performed for $\mathbf{1}$ based on the structures and optical properties determined by single-crystal X-ray diffraction analysis. The calculation was performed with the CASTEP code ${ }^{6}$ based on density functional theory using a plane-wave expansion of the wave functions. The total energy was calculated within the framework of a nonlocal gradient-corrected approximation, the Perdew-Burke-Ernzerhof (PBE) functional. ${ }^{7}$ The interactions between the ionic cores and electrons are described by the norm-conserving pseudopotential. ${ }^{8,9}$ The orbital electrons of $\mathrm{Ba} 5 \mathrm{~s}^{2} 5 \mathrm{p}^{6} 6 \mathrm{~s}^{2}$, Ge $3 \mathrm{~d}^{10} 4 \mathrm{~s}^{2} 4 \mathrm{p}^{2}$, O $2 s^{2} 2 p^{4}$, and Se $3 d^{10} 4 s^{2} 4 p^{4}$ were treated as valence electrons. The number of plane waves included on this basis was determined using a cutoff energy of $800 \mathrm{eV}$, and the numerical integration of the Brillouin zone was performed using a $3 \times 2 \times 1$ Monkhorst-Pack k-point sampling. In the calculation of optical properties and density of states (DOS) of $\mathbf{1}$, more than 101 empty bands and scissors operators of $0.5 \mathrm{eV}$ were applied. Complex dielectric function $\varepsilon(\omega)=\varepsilon_{1}(\omega)+\mathrm{i} \varepsilon_{2}(\omega)$ were calculated, of which the imaginary part $\varepsilon_{2}(\omega)$ generated other optical constants via the Kramers-Kroning transform. ${ }^{10,11}$ The static and dynamic second-order nonlinear susceptibilities $\chi_{\mathrm{abc}}(-2 \omega, \omega, \omega)$ were calculated based on the so-called length-gauge formalism by Aversa and Slipe. ${ }^{12,13}$ The calculated real part $\varepsilon_{1}(\omega)$ and 
the imaginary part $\varepsilon_{2}(\omega)$ of the frequency-dependent optical dielectric functions of $\mathbf{1}$ are illustrated in Figures S10 and S11. It can be found from the dispersion of the calculated $\varepsilon_{2}(\omega)$ spectra that the maximum absorption peaks are located at about 5.8, 6.0 and $6.5 \mathrm{eV}$, which are mainly contributed by the charge transfers from Se $3 p$ and $\mathrm{O} 2 \mathrm{p}$ states to $\mathrm{Ba} 6 \mathrm{~s}$, Ge $4 \mathrm{~s}$ and $\mathrm{Se}$ $4 \mathrm{p}$ states, according to the DOS analysis. The refractive index $n$ and birefringence $\Delta n$ were obtained according to the formulas. ${ }^{14}$ As shown in Figure S13 the static refractive indices are $2.18,2.33$ and 2.27. 


\section{Supporting graphics:}

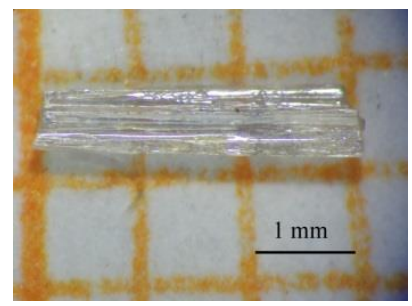

Figure S1. Photo of a medium-size crystal of 1 grown using the facile synthetic method.

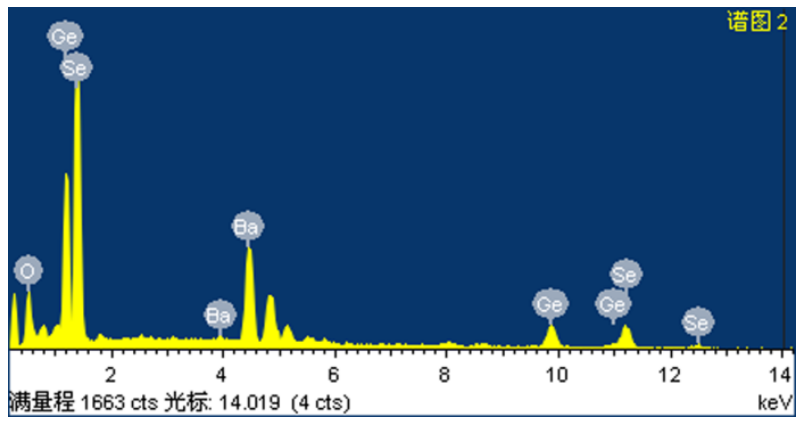

Figure S2. EDX analysis of 1 gives the $\mathrm{Ba} / \mathrm{Ge} / \mathrm{Se}$ molar ratio of 1.1:1.0:2.0.

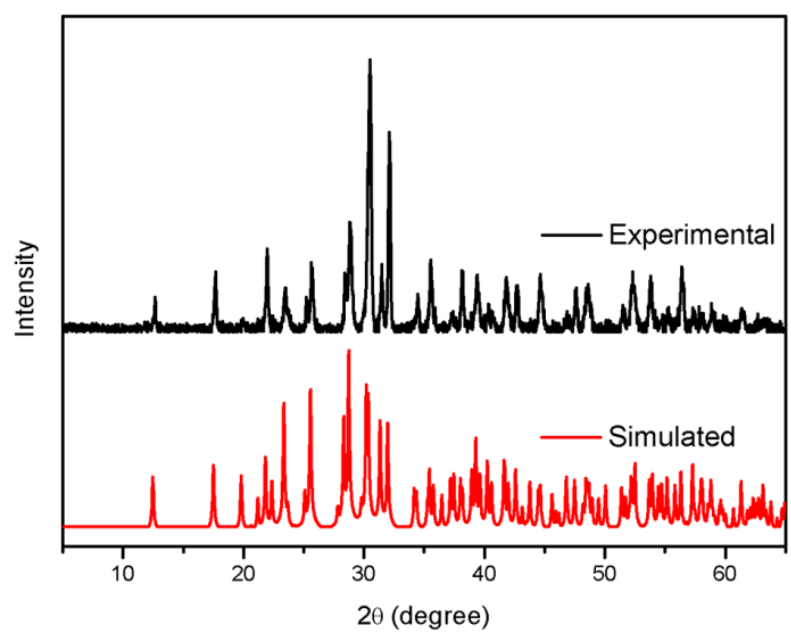

Figure S3. Experimental (top) and Simulated (bottom) powder X-ray diffraction (PXRD) data of 1 . 


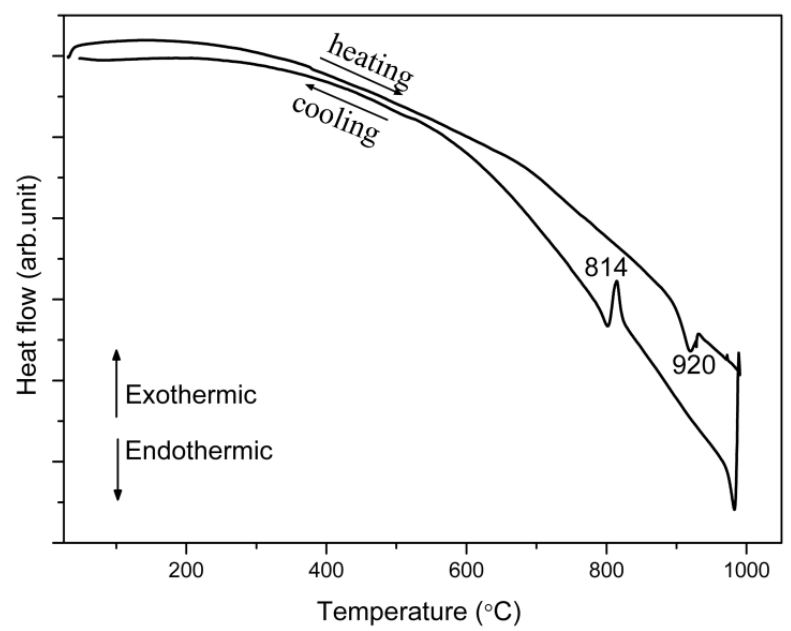

Figure S4. DSC curves reveal the melting and recrystallization events of $\mathbf{1}$.

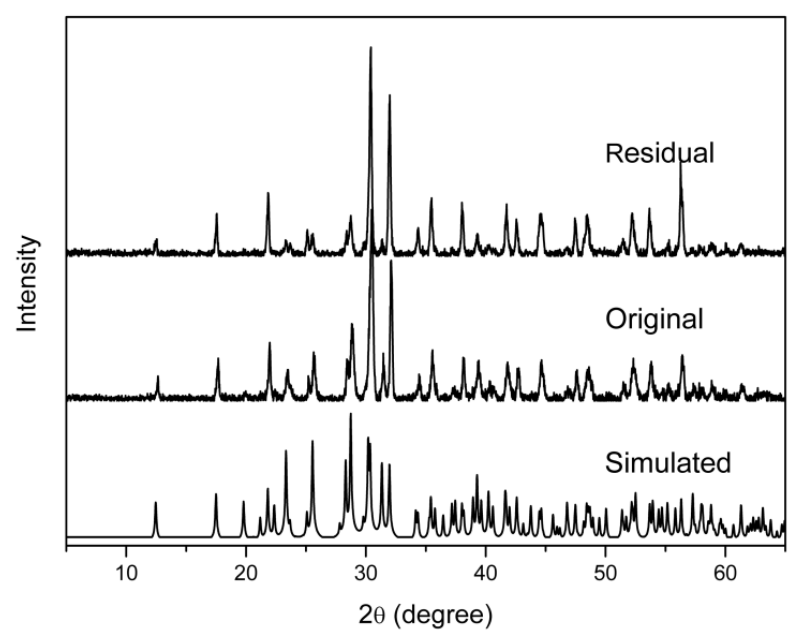

Figure S5. PXRD of the pristine material and the sample obtained after DTA.

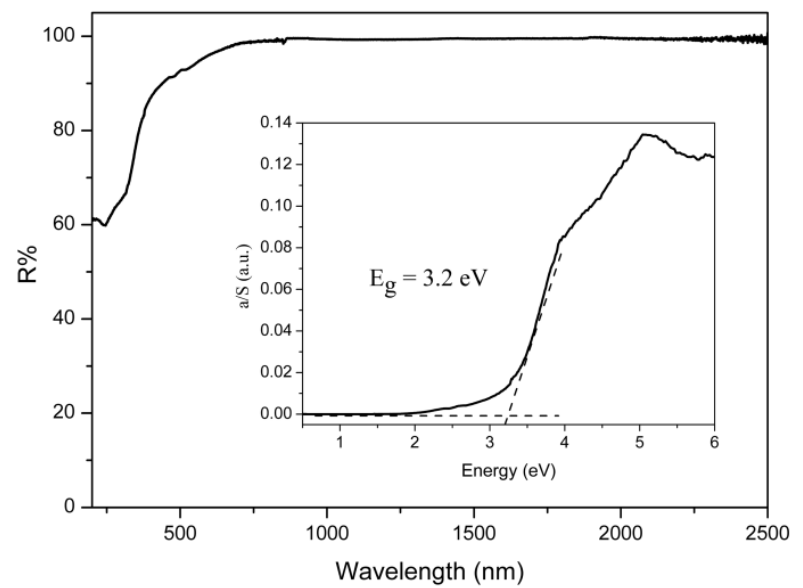

Figure S6. UV-Vis diffuse reflectance spectra of $\mathbf{1}$. 


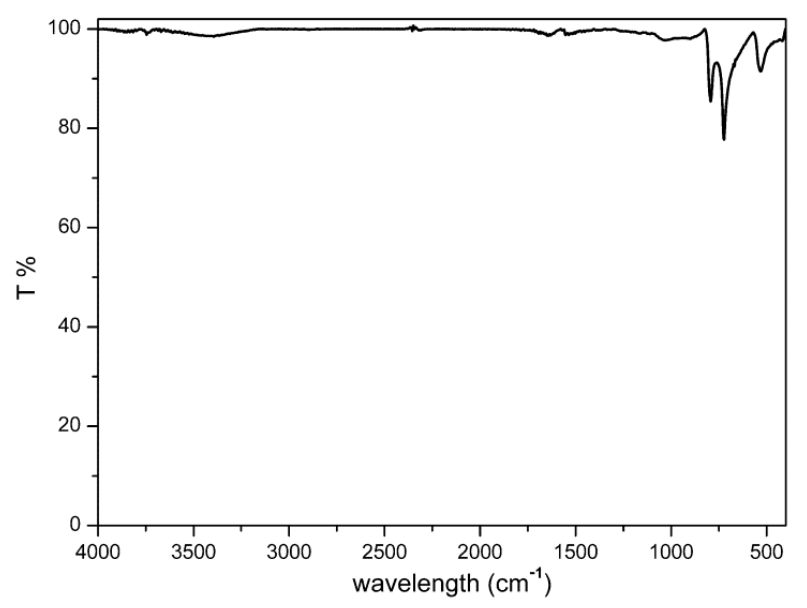

Figure S7. IR spectrum of 1.

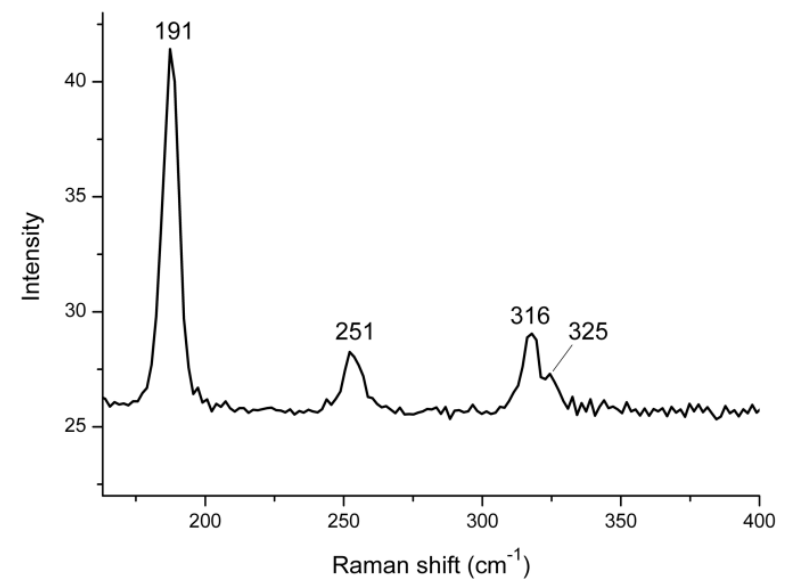

Figure S8. Raman spectrum of $\mathbf{1}$.

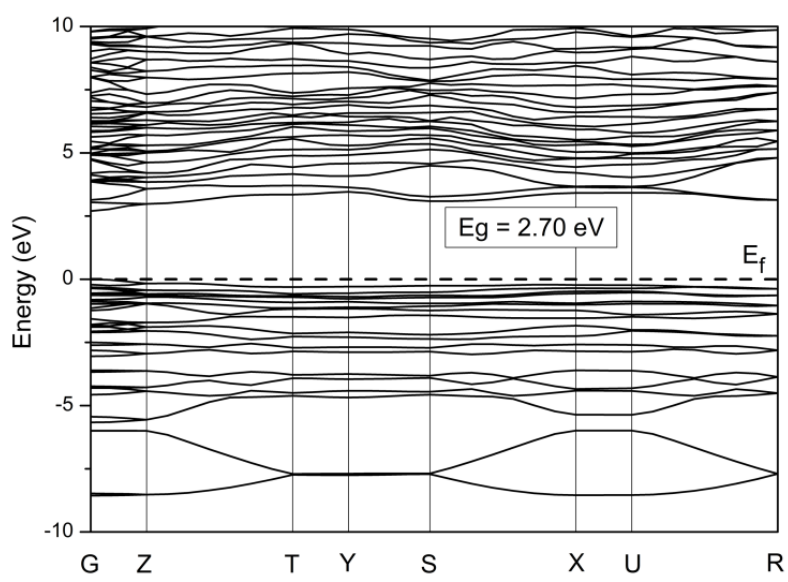

Figure S9. Band structures of $\mathbf{1}$. 


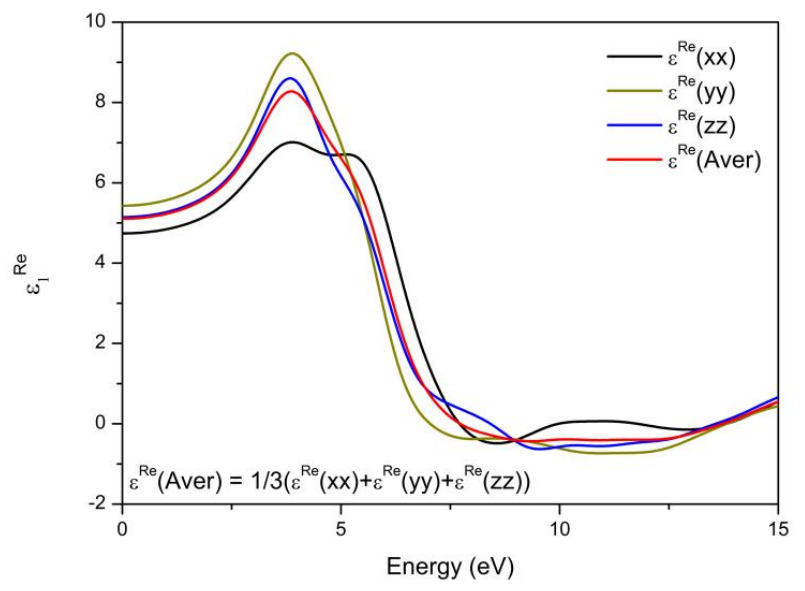

Figure S10. Energy dependences of the real part $\varepsilon_{1}$ of $\mathbf{1}$.

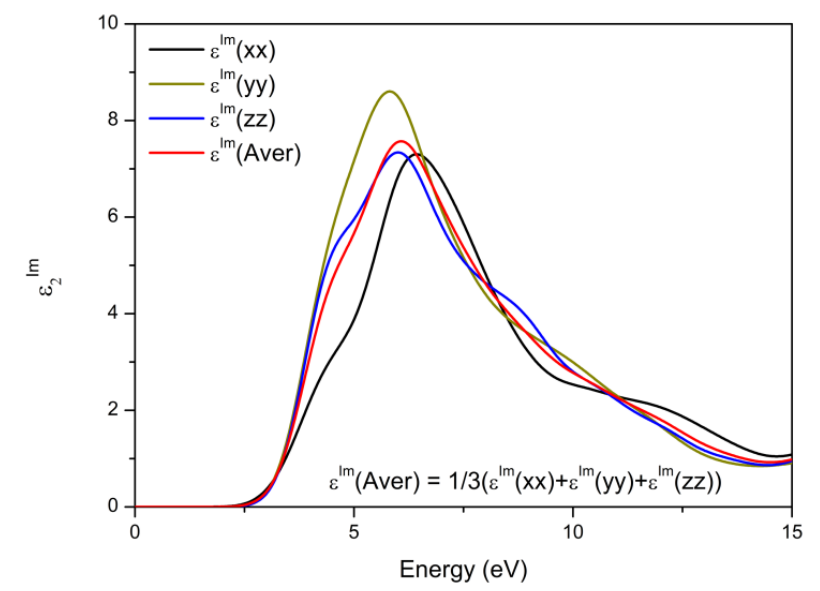

Figure S11. Energy dependences of the imaginary part $\varepsilon_{2}$ of $\mathbf{1}$.

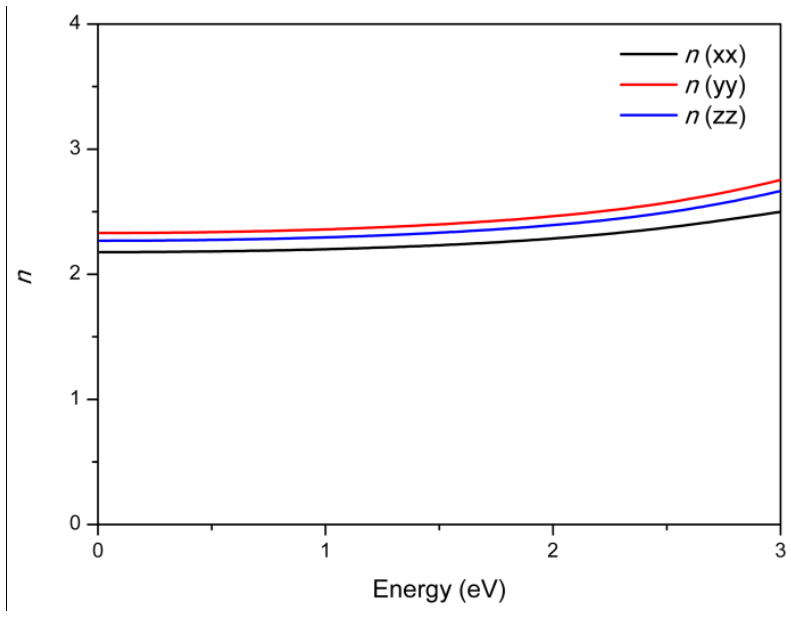

Figure S12. Variations of the calculated refractive index $n$ of $\mathbf{1}$. 


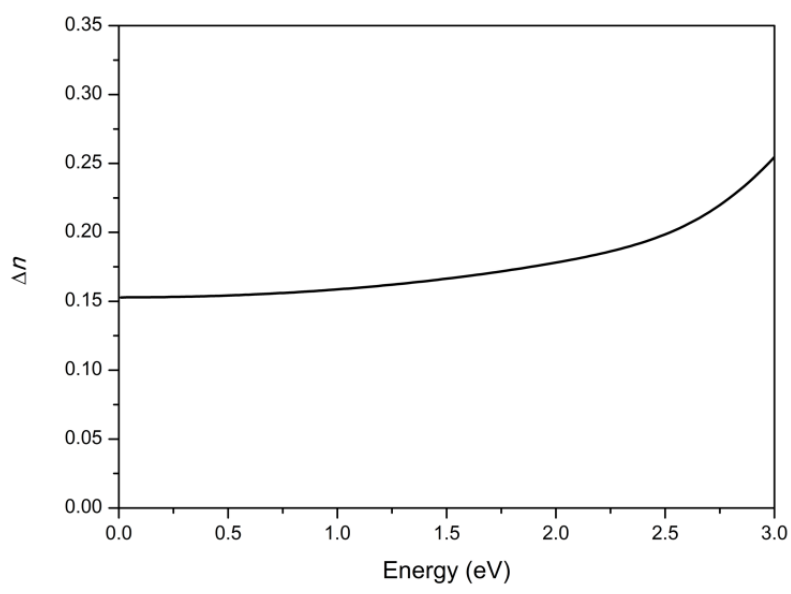

Figure S13. Calculated birefringence $\Delta n$ of 1.

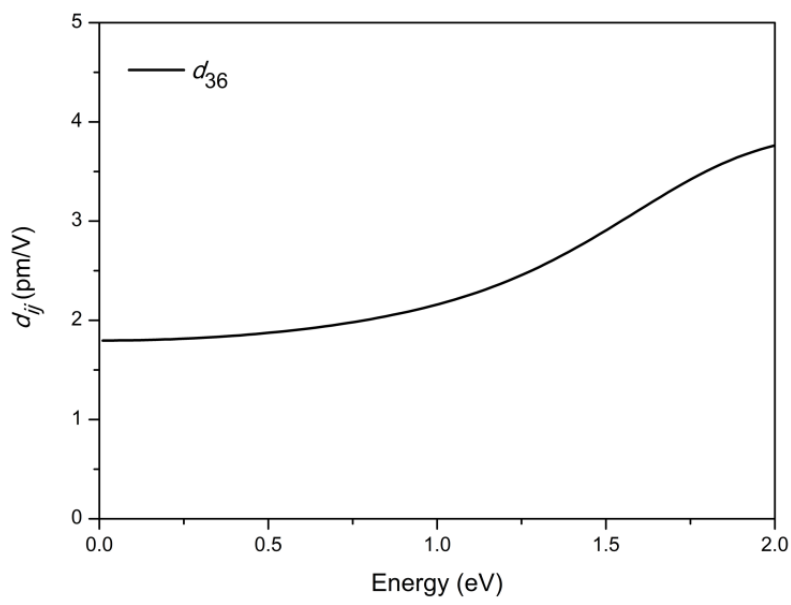

Figure S14. Calculated frequency-dependent SHG coefficients of $\mathbf{1}$. 


\section{Supporting Tables:}

Table S1. Crystal Data and Structure Refinements for $\mathbf{1 .}$

\begin{tabular}{ll}
\hline empirical formula & $\mathrm{BaGeOSe}_{2}(\mathbf{1})$ \\
\hline $\mathrm{fw}$ & 383.85 \\
Space group & $P 2_{1} 2_{1} 2_{1}$ \\
$a(\AA)$ & $4.798(2)$ \\
$b(\AA)$ & $8.609(5)$ \\
$c(\AA)$ & $12.524(7)$ \\
$V\left(\AA^{3}\right)$ & $517.3(5)$ \\
$\mathrm{Z}$ & 4 \\
$\mathrm{D}_{\text {calcd }}\left(\mathrm{g} \cdot \mathrm{m}^{-3}\right)$ & 4.929 \\
$\mathrm{Flack}$ parameter $x$ & $0.00(2)$ \\
$R_{1}{ }^{a}(I>2 \sigma(I))$ & 0.0196 \\
$w R_{2}{ }^{\mathrm{b}}(I>2 \sigma(I))$ & 0.0372 \\
$R_{1}{ }^{a}($ all data $)$ & 0.0202 \\
$w R_{2}{ }^{b}$ (all data) & 0.0374 \\
$\mathrm{GOF}$ on $F^{2}$ & 1.047 \\
$\Delta \rho_{\text {max }} / \Delta \rho_{\text {min, }}\left(\mathrm{e} \AA^{-3}\right)$ & $0.739 /-0.675$ \\
${ }^{a} R I=\Sigma|| F_{o}|-| F_{c}|| / \Sigma\left|F_{o}\right|,{ }^{b} w R 2=\left(\Sigma\left(w\left(F_{o}{ }^{2}-F_{c}{ }^{2}\right)^{2}\right) / \Sigma\left(w\left(F_{o}{ }^{2}\right)^{2}\right)\right)^{1 / 2}$. \\
\hline
\end{tabular}

Table S2. Selected Bond Distances ( $)$ and Angles (deg) in $\mathbf{1}$.

\begin{tabular}{|c|c|c|c|}
\hline \multicolumn{4}{|c|}{ Bond Distances $(\AA)$} \\
\hline $\mathrm{Ba}(1)-\mathrm{O}(1)$ & $2.608(4)$ & $\mathrm{Ba}(1)-\mathrm{Se}(2)$ & $3.616(1)$ \\
\hline $\mathrm{Ba}(1)-\mathrm{Se}(1)$ & $3.283(1)$ & $\mathrm{Ge}(1)-\mathrm{O}(1)$ & $1.789(4)$ \\
\hline $\mathrm{Ba}(1)-\mathrm{Se}(1)$ & $3.328(1)$ & $\mathrm{Ge}(1)-\mathrm{O}(1)$ & $1.790(4)$ \\
\hline $\mathrm{Ba}(1)-\mathrm{Se}(1)$ & $3.336(1)$ & $\mathrm{Ge}(1)-\mathrm{Se}(2)$ & $2.291(1)$ \\
\hline $\mathrm{Ba}(1)-\mathrm{Se}(2)$ & $3.408(2)$ & $\mathrm{Ge}(1)-\mathrm{Se}(1)$ & $2.305(1)$ \\
\hline $\mathrm{Ba}(1)-\mathrm{Se}(2)$ & $3.416(1)$ & & \\
\hline \multicolumn{4}{|c|}{ Bond Angles (deg) } \\
\hline $\mathrm{O}(1)-\mathrm{Ba}(1)-\mathrm{Se}(1)$ & $135.82(8)$ & $\mathrm{Se}(2)-\mathrm{Ba}(1)-\mathrm{Se}(2)$ & $73.87(2)$ \\
\hline $\mathrm{O}(1)-\mathrm{Ba}(1)-\mathrm{Se}(1)$ & $130.56(8)$ & $\mathrm{O}(1)-\mathrm{Ba}(1)-\mathrm{Se}(2)$ & $68.44(8)$ \\
\hline $\operatorname{Se}(1)-\mathrm{Ba}(1)-\mathrm{Se}(1)$ & $93.07(4)$ & $\mathrm{Se}(1)-\mathrm{Ba}(1)-\mathrm{Se}(2)$ & $78.82(4)$ \\
\hline $\mathrm{O}(1)-\mathrm{Ba}(1)-\mathrm{Se}(1)$ & $62.44(9)$ & $\mathrm{Se}(1)-\mathrm{Ba}(1)-\mathrm{Se}(2)$ & $145.46(2)$ \\
\hline $\mathrm{Se}(1)-\mathrm{Ba}(1)-\mathrm{Se}(1)$ & $82.58(4)$ & $\mathrm{Se}(1)-\mathrm{Ba}(1)-\mathrm{Se}(2)$ & $77.58(3)$ \\
\hline $\mathrm{Se}(1)-\mathrm{Ba}(1)-\mathrm{Se}(1)$ & $135.14(3)$ & $\mathrm{Se}(2)-\mathrm{Ba}(1)-\mathrm{Se}(2)$ & $71.37(2)$ \\
\hline $\mathrm{O}(1)-\mathrm{Ba}(1)-\mathrm{Se}(2)$ & $122.13(9)$ & $\mathrm{Se}(2)-\mathrm{Ba}(1)-\mathrm{Se}(2)$ & $86.00(4)$ \\
\hline $\mathrm{Se}(1)-\mathrm{Ba}(1)-\mathrm{Se}(2)$ & $70.28(3)$ & $\mathrm{O}(1)-\mathrm{Ge}(1)-\mathrm{O}(1)$ & $104.45(8)$ \\
\hline $\mathrm{Se}(1)-\mathrm{Ba}(1)-\mathrm{Se}(2)$ & $74.28(3)$ & $\mathrm{O}(1)-\mathrm{Ge}(1)-\mathrm{Se}(2)$ & $104.79(12)$ \\
\hline $\mathrm{Se}(1)-\mathrm{Ba}(1)-\mathrm{Se}(2)$ & $141.85(3)$ & $\mathrm{O}(1)-\mathrm{Ge}(1)-\mathrm{Se}(2)$ & $109.68(13)$ \\
\hline $\mathrm{O}(1)-\mathrm{Ba}(1)-\mathrm{Se}(2)$ & $63.58(8)$ & $\mathrm{O}(1)-\mathrm{Ge}(1)-\mathrm{Se}(1)$ & $111.97(13)$ \\
\hline
\end{tabular}




\begin{tabular}{|l|l|l|l|}
\hline $\mathrm{Se}(1)-\mathrm{Ba}(1)-\mathrm{Se}(2)$ & $143.89(2)$ & $\mathrm{O}(1)-\mathrm{Ge}(1)-\mathrm{Se}(1)$ & $99.50(12)$ \\
\hline $\mathrm{Se}(1)-\mathrm{Ba}(1)-\mathrm{Se}(2)$ & $81.17(4)$ & $\mathrm{Se}(2)-\mathrm{Ge}(1)-\mathrm{Se}(1)$ & $124.77(3)$ \\
\hline $\mathrm{Se}(1)-\mathrm{Ba}(1)-\mathrm{Se}(2)$ & $125.93(2)$ & & \\
\hline
\end{tabular}

\section{References}

(1) CrystalClear, version 1.3.5; Rigaku Corp.: Tokyo, 2002.

(2) SHELXTL Reference Manual, version 5; Siemens Energy \& Automation Inc.: Madison, WI, 1994.

(3) Spek, A. L. PLATON, A Multipurpose Crystallographic Tool; Utrecht University: Utrecht, The Netherlands, 2005.

(4) Gelato, L. M.; Parthé, E. STRUCTURE TIDY- a computer program to standardize crystal structure data. J. Appl. Crystallogr. 1987, 20, 139-143.

(5) Korum, G. Reflectance Spectroscopy; Springer: New York, 1969.

(6) Segall, M. D.; Lindan, P. J. D.; Probert, M. J.; Pickard, C. J.; Hasnip, P. J.; Clark S. J.; M. Payne, C. First-principles simulation: ideas, illustrations and the CASTEP code. J. Phys.: Condens. Matter, 2002, 14, $2717-2744$.

(7) Perdew, J. P.; Burke K.; Ernzerhof, M. Generalized gradient approximation made simple. Phys. Rev. Lett. 1996, 77, 3865-3868.

(8) (a) Hamann, D. R.; Schluter M.; Chiang, C. Norm-Conserving Pseudopotentials. Phys. Rev. Lett. 1979, $43,1494-1497$.

(9) Lin, J. S.; Qteish, A.; Payne, M. C.; Heine, V. Optimized and transferable nonlocal separable ab initio pseudopotentials. Phys. Rev. B 1993, 47, 4174-4180.

(10) Laksari, S.; Chahed, A.; Abbouni, N.; Benhelal, O.; Abbar, B. First-principles calculations of the structural, electronic and optical properties of $\mathrm{CuGaS}_{2}$ and $\mathrm{AgGaS}_{2}$. Comput. Mater. Sci. 2006, 38, $223-230$.

(11) Mo, S. D.; Ching, W. Y. Electronic and optical properties of three phases of titanium dioxide: Rutile, anatase, and brookite. Phys. Rev. B: Condens. Matter. 1995, 51, 13023-13032.

(12) (a) Aversa , C.; Sipe, J. E. Nonlinear optical susceptibilities of semiconductors: Results with a 
length-gauge analysis. Phys. Rev. B: Condens. Matter, 1995, 52, 14636-14645.

(13) Rashkeev, S. N.; Lambrecht, W. R. L.; Segall, B. Second-harmonic generation in SiC polytypes. Phys. Rev. B: Condens. Matter, 1998, 57, 3905-3919.

(14) Yu, P.; Zhou L. J.; Chen. L. Noncentrosymmetric inorganic open-framework chalcohalides with strong middle IR SHG and red emission: $\mathrm{Ba}_{3} \mathrm{AGa}_{5} \mathrm{Se}_{10} \mathrm{Cl}_{2}(\mathrm{~A}=\mathrm{Cs}, \mathrm{Rb}, \mathrm{K})$. J. Am. Chem. Soc. 2012, 134, $2227-2235$. 